\title{
Approximation of Smectic-A Liquid CRYstals
}

\author{
Francisco Guillén-GonzÁlez* Giordano Tierra ${ }^{\dagger}$
}

\begin{abstract}
In this paper, we present energy-stable numerical schemes for a Smectic-A liquid crystal model. This model involve the hydrodynamic velocity-pressure macroscopic variables $(\boldsymbol{u}, p)$ and the microscopic order parameter of Smectic-A liquid crystals, where its molecules have a uniaxial orientational order and a positional order by layers of normal and unitary vector $\boldsymbol{n}$.

We start from the formulation given in [E'97 by using the so-called layer variable $\phi$ such that $\boldsymbol{n}=\nabla \phi$ and the level sets of $\phi$ describe the layer structure of the Smectic-A liquid crystal. Then, a strongly non-linear parabolic system is derived coupling velocity and pressure unknowns of the Navier-Stokes equations $(\boldsymbol{u}, p)$ with a fourth order parabolic equation for $\phi$.

We will give a reformulation as a mixed second order problem which let us to define some new energy-stable numerical schemes, by using second order finite differences in time and $C^{0}$ finite elements in space. Finally, numerical simulations are presented for $2 D$-domains, showing the evolution of the system until it reachs an equilibrium configuration.

Up to our knowledge, there is not any previous numerical analysis for this type of models.
\end{abstract}

Key words: Liquid crystal, micro-macro model, second order time scheme, finite elements, energy stability

Mathematics subject classifications (1991): 35Q35, 65M60, 76A15

\section{Introduction}

The topic of Liquid Crystals (LCs) is a multidisciplinary field related to Chemistry, Engineering, Biology, Medicine, Physics and Mathematics. Usually, the discovery of liquid crystals is attributed to botanist F. Reinitzer who in 1888 found a substance that appeared to have two different melting points. A year later, O. Lehmann solved the problem with the description of a new state of matter midway between a liquid and a crystal. In 1922, G. Friedel spoke for the first time about mesophases and in 1991 Pierre-Gilles de Gennes was awarded with Nobel Prize in Physics for his contributions related to LCs, in particular, for discovering that "methods developed for studying order phenomena in simple systems can be generalized to more complex forms of matter, in particular to liquid crystals and polymers". LCs are a state of matter that can be viewed as intermediate phases between solids and isotropic fluids. Indeed, macroscopically such materials may flow like fluids but at microscopic scale their molecules have orientational properties (due to elasticity effects) and they can experience deformations as elastic solids, hence LCs can be considered as anisotropic liquids.

Furthermore, LCs can be divided into thermotropic and lyotropic phases, where thermotropic phases change their state as the temperature is varying while lyotropic phase change of state as concentration is varying.

\footnotetext{
*Departamento de Ecuaciones Diferenciales y Análisis Numérico, Universidad de Sevilla, Aptdo. 1160, 41080 Seville, Spain, email: guillen@us.es

${ }^{\dagger}$ Mathematical Institute, Faculty of Mathematics and Physics, Charles University, Prague 8, 186 75, Czech Republic, e-mail: gtierra@karlin.mff.cuni.cz
} 
Examples of LCs can be widely found in the natural world and in technological applications. For instance, most contemporary electronic devices use LCs for their displays and lyotropic liquidcrystalline phases can be found in living systems, forming proteins and cell membranes.

Within thermotropic LCs can be distinguished into two main different phases: Nematic and Smectic (see Figure 1.1 for schematic description of Nematic and Smectic phases). In Nematic phases, the rodshaped molecules have no positional order, but molecules self-align to have a long-range directional order with their long axes roughly parallel. Thus, the molecules are free to flow and their center of mass positions are randomly distributed as in a liquid, although they still maintain their long-range directional order. Moreover, most Nematics are uniaxial, i.e., they have one axis that is longer and preferred.

In Smectic phases (which are found at lower temperatures than the Nematic ones) molecules form well-defined layers that can slide over one another, i.e., Smectics are thus positionally ordered along one direction and are liquid-like within the layers. In particular, in Smectic-A phases, molecules are oriented along the normal vector of the layer, while in Smectic-C phases they are tilted away from the normal vector of the layer. We refer the reader to Collings'90 for further information on the physics and properties of the different LCs that can be found in the nature.

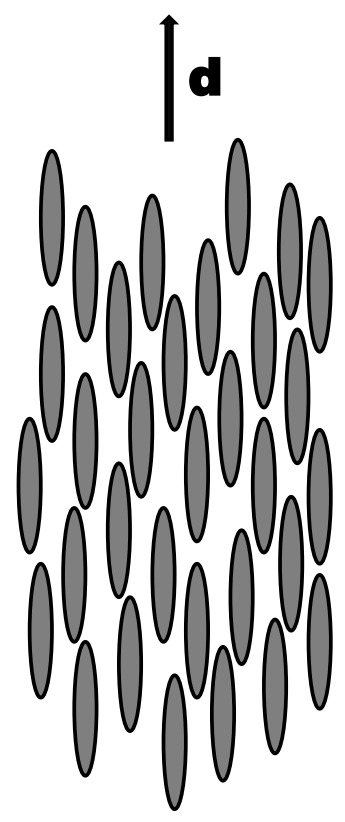

Nematic

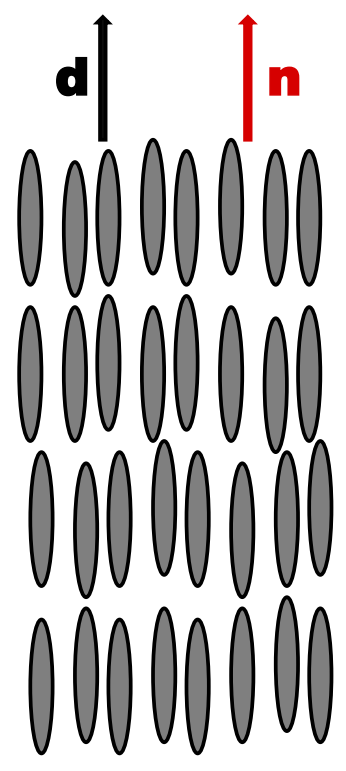

Smectic-A

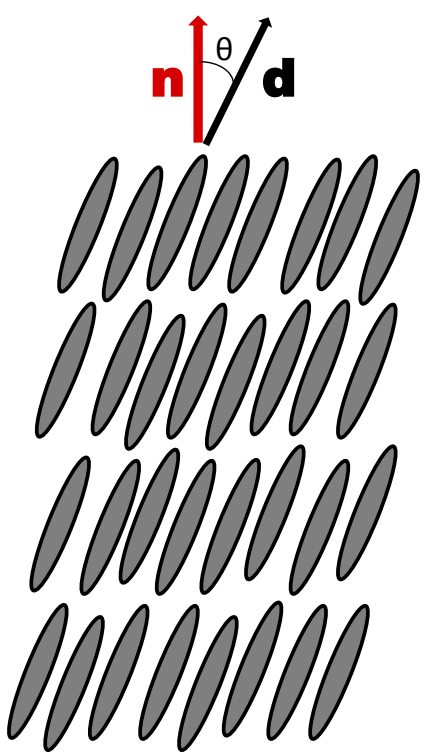

Smectic-C

Figure 1.1: Different phases of Liquid Crystals

The dynamics interaction between the macroscopic level and the microscopic order of molecules are modeled with (strongly nonlinear) parabolic PDE systems, involving:

- the macroscopic fluid dynamics in velocity-pressure variables of the Navier-Stokes type,

- a microscopic order variable modeled by a (vectorial) gradient flow system. 
We assume LCs confined in an open bounded domain $\Omega \subset \mathbb{R}^{N}(\mathrm{~N}=2$ or 3$)$ with boundary $\partial \Omega$ which is thermally isolated during the time interval $[0,+\infty)$. Then, the macroscopic dynamic can be described by the velocity-pressure variables $\boldsymbol{u}: \Omega \times[0,+\infty) \rightarrow \mathbb{R}^{N}$ and $p: \Omega \times[0,+\infty) \rightarrow \mathbb{R}$ respectively. For isotropic fluids, these variables are governed by the Navier-Stokes equations, but in LCs the anisotropic microscopic configurations modify the macroscopic dynamics, and reciprocally, the macroscopic movement has an influence on the orientation of molecules.

The mathematical systems related with LCs have been widely study in recent times, where most of these works are devoted to study Nematic phases. A simplified model for Nematic LCs was introduced by Lin in [Lin'89] and studied by Lin and Liu in [Lin-Liu'95, Lin-Liu'00] and by Coutand and Shkoller in Coutand-Shkoller'01. A simplified model for Smectic-A LC was proposed by E in [E'97] and studied by Liu in [Liu'00, by Climent and Guillén in [Climent-Guillen'10, Climent-Guillen'14] and by $\mathrm{Wu}$ and Segatti in [Wu-Segatti]. Both are simplified models from the original equations in the continuum theory of liquid crystals due to Ericksen and Leslie, which was developed during the period of 1958 through 1968. We refer the reader to Climent-Guillen'13 for a detailed review about the main results in the mathematical analysis of these models.

From the numerical analysis and simulations point of view, most of the effort of researchers have been focused on approximating Nematic phases. We recommend the reader [Badia-Guillén-Gutiérrez'11, Tierra-Guillen'14 (and the references therein) as state-of-the-art reviews of numerical schemes to approximate Nematic LCs and other energy-based models. For Smectic phases, the literature concerning the development of numerical schemes (and the study of their properties) is still very scarce. The main concern of this paper is to fulfill this lack of numerical approximations of Smectic phases, presenting for the first time energy-stable numerical schemes to approximate these systems.

This work is organized as follows: In Section 2, we recall the theory of Smectic-A, starting with the static Oseen-Frank's theory and then detailing the dynamics of these systems. Afterwards, we introduce a reformulation of the system that allows the spatial approximation of the system considering $C^{0}$ finite elements.

Then, numerical schemes to approximate this reformulation of the system are presented in Section 3. We first introduce a finite element scheme for the spatial approximation and afterwards we present a generic second order finite differences approximation in time.

We have carried out numerical simulations to study the performance of the proposed schemes. In particular, we have detailed the results of these computations in Section 4. Finally, we state the conclusion of our work in Section 5 .

\section{Smectic-A Liquid Crystals}

\subsection{Static Oseen-Frank's theory of Smectic-A LCs}

Let $\Omega \subset \mathbb{R}^{d}(d=2,3)$ be the bounded domain occupied by the LC, with boundary $\partial \Omega$. The equilibrium states correspond to a minimum of a (elastic) free energy governing the system. An usual form for this energy is the Oseen-Frank free energy:

$$
E_{\text {ela }}(\boldsymbol{d}, \nabla \boldsymbol{d})=\int_{\Omega}\left(\frac{k_{1}}{2}(\nabla \cdot \boldsymbol{d})^{2}+\frac{k_{2}}{2}(\boldsymbol{d} \cdot(\nabla \times \boldsymbol{d}))^{2}+\frac{k_{3}}{2}|\boldsymbol{d} \times(\nabla \times \boldsymbol{d})|^{2}\right) d \boldsymbol{x},
$$

where $\boldsymbol{d}=\boldsymbol{d}(x)$ is the director vector and $k_{i}>0$ are elastic constants (hereafter, $|\cdot|$ denotes the euclidean norm). A further simplification is the equal constant case $k_{1}=k_{2}=k_{3}(=1)$, where $E_{\text {ela }}$ reduces to the Dirichlet energy functional:

$$
E_{\text {ela }}=\frac{1}{2} \int_{\Omega}(\nabla \cdot \boldsymbol{d})^{2} d \boldsymbol{x} .
$$


For uniaxial Smectic LCs, the molecules are arranged in almost incompressible layers (whose normal vector is denoted by $\boldsymbol{n}$ ) of almost constant width. Moreover, each layer consists of a single optical axis $\boldsymbol{d}$ perpendicular to the layer. In particular, for Smectic-A LCs, both unitary vectors coincide

$$
\boldsymbol{d}=\boldsymbol{n} .
$$

On the other hand, due to the incompressibility of the layers

$$
\nabla \times \boldsymbol{n}=0,
$$

there exists a potential function $\phi: \Omega \rightarrow \mathbb{R}$ such that

$$
\boldsymbol{n}=\nabla \phi
$$

and the level sets of $\phi$ will represent the layer structure. Moreover, if the domain $\Omega$ has a simply connected boundary $\partial \Omega$, then $\phi$ can be computed via the Poisson-Neumann problem:

$$
-\Delta \phi=-\nabla \cdot \boldsymbol{n} \quad \text { in } \Omega, \quad \nabla \phi \cdot \boldsymbol{m}=\boldsymbol{n} \cdot \boldsymbol{m} \quad \text { on } \partial \Omega,
$$

where $\boldsymbol{m}$ denotes the exterior normal vector to $\partial \Omega$.

Since $\boldsymbol{d}=\boldsymbol{n}, \nabla \times \boldsymbol{n}=0$ and $\boldsymbol{n}=\nabla \phi$, the (elastic) Dirichlet functional can be reduced to

$$
E_{\text {ela }}=\frac{1}{2} \int_{\Omega}(\nabla \cdot \boldsymbol{n})^{2} d \boldsymbol{x}=\frac{1}{2} \int_{\Omega}(\Delta \phi)^{2} d \boldsymbol{x}
$$

and the static minimization problem has a convex functional but with a non-convex constraint:

$$
\min _{\phi} E_{\text {ela }}(\Delta \phi) \quad \text { subject to } \quad|\nabla \phi|=1 .
$$

The optimality system associates to this minimization problem reads:

$$
\frac{\delta E_{\text {ela }}}{\delta \phi}:=\Delta^{2} \phi-\nabla \cdot(\xi \nabla \phi)=0, \quad|\nabla \phi|=1,
$$

where $\xi$ is a Lagrange multiplier. In order to relax the non-convex constraint $|\nabla \phi|=1$, it is usual to consider the following regularized energy (by considering a penalization of the Ginzburg-Landau type):

$$
E(\phi)=E_{\text {ela }}(\Delta \phi)+E_{\text {pen }}(\nabla \phi):=\frac{1}{2} \int_{\Omega}|\Delta \phi|^{2}+\frac{1}{\varepsilon^{2}} \int_{\Omega} F(\nabla \phi), \quad F(\boldsymbol{n})=\frac{1}{4}\left(|\boldsymbol{n}|^{2}-1\right)^{2} .
$$

Then the relaxed minimization problem is now a problem without constraints but for a non-convex functional:

$$
\min _{\phi} E(\phi) .
$$

The optimality system associated to this problem reads

$$
\left\langle\frac{\delta E(\phi)}{\delta \phi}\right\rangle=\int_{\Omega} \Delta \phi \Delta \bar{\phi}+\frac{1}{\varepsilon^{2}} \boldsymbol{f}(\nabla \phi) \cdot \nabla \bar{\phi}=0 \quad \forall \bar{\phi}
$$

where $\boldsymbol{f}(\boldsymbol{n})=\nabla_{\boldsymbol{n}} F(\boldsymbol{n})=\left(|\boldsymbol{n}|^{2}-1\right) \boldsymbol{n}$. In order to show which boundary conditions are admissible, we need to split the computations into two different steps: 
1. Assume $\left.\Delta \phi\right|_{\partial \Omega}=0[\mathrm{~N} 1]$ or $\left.\nabla \bar{\phi} \cdot \boldsymbol{m}\right|_{\partial \Omega}=0[\mathrm{D} 2]$, arriving at

$$
\left\langle\frac{\delta E(\phi)}{\delta \phi}\right\rangle=-\int_{\Omega} \boldsymbol{w} \cdot \nabla \bar{\phi}, \quad \boldsymbol{w}:=\nabla \Delta \phi-\frac{1}{\varepsilon^{2}} \boldsymbol{f}(\nabla \phi) .
$$

2. Then, assume $\left.\boldsymbol{w} \cdot \boldsymbol{m}\right|_{\partial \Omega}=0[\mathrm{~N} 2]$ or $\left.\bar{\phi}\right|_{\partial \Omega}=0[\mathrm{D} 1]$, to arrive at

$$
\frac{\delta E(\phi)}{\delta \phi}=\nabla \cdot \boldsymbol{w}=\Delta^{2} \phi-\frac{1}{\varepsilon^{2}} \nabla \cdot \boldsymbol{f}(\nabla \phi) \quad \text { in } \Omega .
$$

Therefore, the admissible combinations for boundary conditions are:

$$
\left\{\begin{array}{lll}
{[\mathrm{D} 1-\mathrm{D} 2]} & \left.\phi\right|_{\partial \Omega}=\phi_{1}, & \left.\nabla \phi \cdot \boldsymbol{m}\right|_{\partial \Omega}=\phi_{2}, \\
{[\mathrm{D} 1-\mathrm{N} 1]} & \left.\phi\right|_{\partial \Omega}=\phi_{1}, & \left.\Delta \phi\right|_{\partial \Omega}=0, \\
{[\mathrm{D} 2-\mathrm{N} 2]} & \left.\nabla \phi \cdot \boldsymbol{m}\right|_{\partial \Omega}=\phi_{2}, & \left.\boldsymbol{w} \cdot \boldsymbol{m}\right|_{\partial \Omega}=0 \\
{[\mathrm{~N} 1-\mathrm{N} 2]} & \left.\Delta \phi\right|_{\partial \Omega}=0, & \left.\boldsymbol{w} \cdot \boldsymbol{m}\right|_{\partial \Omega}=0 .
\end{array}\right.
$$

Note that pairs [D1-N2] and [D2-N1] are not admissible boundary conditions in order to get (2.14).

\subsection{Dynamics of Smectic-A LCs.}

We are interested in the dynamics of Smectic-A LCs confined in the domain $\Omega$ during a time interval $(0, T)$.

The macroscopic dynamic variables are denoted by $(\boldsymbol{u}, p)$, that represents the incompressible velocity and the pressure of the flow, respectively. For the well known case of isotropic newtonian fluids (assuming constant density), the equilibrium of forces is modeled in terms of these macroscopic variables $(\boldsymbol{u}, p)$, arriving at the PDE system (linear momentum system):

$$
\frac{D \boldsymbol{u}}{D t}-\nabla \cdot \sigma=0, \quad \nabla \cdot \boldsymbol{u}=0 \quad \text { in } \Omega \times(0, T),
$$

where $\frac{D \boldsymbol{u}}{D t}=\boldsymbol{u}_{t}+(\boldsymbol{u} \cdot \nabla) \boldsymbol{u}$ is the convective time derivative (the derivative along the streamlines), $\sigma$ is the Cauchy stress tensor given by the so-called Stokes' law:

$$
\sigma=-p \mathcal{I}+2 \nu D(\boldsymbol{u})
$$

where $\mathcal{I}$ represents the $N$-dimensional identity matrix and $D(\boldsymbol{u})=\left(\nabla \boldsymbol{u}+\nabla \boldsymbol{u}^{t}\right) / 2$ is the deformation tensor (symmetric part of $\nabla \boldsymbol{u}$ ).

For Smectic-A LCs, we will consider the model proposed by E [E'97], where the elastic (and dissipative) influence of the order vector $\boldsymbol{n}$ in the linear momentum system is modeled by the following Cauchy stress tensor:

$$
\sigma=-p \mathcal{I}+\sigma^{d}(D(\boldsymbol{u}), \boldsymbol{n})+\sigma^{e}(\boldsymbol{n}), \quad \boldsymbol{n}=\nabla \phi,
$$

where $\sigma^{d}=\sigma^{d}(D(\boldsymbol{u}), \boldsymbol{n})$ is the dissipative (symmetric) stress tensor defined as:

$$
\sigma^{d}=\mu_{1}\left(\boldsymbol{n}^{t} D(\boldsymbol{u}) \boldsymbol{n}\right) \boldsymbol{n} \otimes \boldsymbol{n}+\mu_{4} D(\boldsymbol{u})+\mu_{5}(D(\boldsymbol{u}) \boldsymbol{n} \otimes \boldsymbol{n}+\boldsymbol{n} \otimes D(\boldsymbol{u}) \boldsymbol{n}),
$$

and $\sigma^{e}$ is the (nonsymmetric) elastic tensor, defined as:

$$
\sigma^{e}=-\nabla \phi \otimes \boldsymbol{w}+\Delta \phi \nabla(\nabla \phi),
$$

where $\boldsymbol{w}$ was defined in $(2.13)$ and $\otimes$ denotes the tensorial product. Then, it is possible to derive a PDE system for Smectic-A LC: 
- Conservation of linear momentum derives to the $(\boldsymbol{u}, p)$-system:

$$
\frac{D \boldsymbol{u}}{D t}+\nabla p-\nabla \cdot\left(\sigma^{d}+\lambda \sigma^{e}\right)=0, \quad \nabla \cdot \boldsymbol{u}=0 \quad \text { in } \Omega \times(0, T),
$$

where $\lambda>0$ is a constant coefficient coupling the kinetic and the elastic energy.

- Conservation of angular momentum derives the $\phi$-equation:

$$
\frac{D \phi}{D t}+\gamma \frac{\delta E}{\delta \phi}=0 \quad \text { in } \Omega \times(0, T),
$$

where $\gamma>0$ is a constant coefficient (elastic relaxation time) and $\frac{\delta E}{\delta \phi}$ is defined in 2.14). Note that this equation can be viewed as a Allen-Cahn type from the phase-field framework.

The elastic tensor $\sigma^{e}(\boldsymbol{n})$ must be chosen in order to assume that the dynamics is governed by the (dissipative) energy equality:

$$
\frac{d}{d t}\left(E_{k i n}(\boldsymbol{u})+\lambda E(\phi)\right)+\int_{\Omega} \sigma^{d}(D(\boldsymbol{u}), \boldsymbol{n}): D(\boldsymbol{u})+\lambda \gamma \int_{\Omega}\left|\frac{\delta E}{\delta \phi}\right|^{2}=0,
$$

where $E_{k i n}(\boldsymbol{u})=\frac{1}{2} \int_{\Omega}|\boldsymbol{u}|^{2}$ is the kinetic energy and

$$
\sigma^{d}(D(\boldsymbol{u}), \boldsymbol{n}): D(\boldsymbol{u})=\mu_{1}\left(\boldsymbol{n}^{t} D(\boldsymbol{u}) \boldsymbol{n}\right)^{2}+\mu_{4}|D(\boldsymbol{u})|^{2}+\mu_{5}|D(\boldsymbol{u}) \boldsymbol{n}|^{2} \geq \mu_{4}|D(\boldsymbol{u})|^{2} .
$$

Assuming time-independent boundary conditions for $\phi$, that is $\left.\phi_{t}\right|_{\partial \Omega}=0$ and $\left.\nabla \phi_{t} \cdot \boldsymbol{m}\right|_{\partial \Omega}=0$, this energy equality is deduced considering

$$
\int_{\Omega}(\boldsymbol{u} \text {-system }) \cdot \boldsymbol{u}+\lambda \int_{\Omega}(\phi \text {-equation }) \frac{\delta E}{\delta \phi},
$$

because the following equality holds [E'97]:

$$
-\nabla \cdot \sigma^{e}=-\frac{\delta E(\phi)}{\delta \phi} \nabla \phi+\nabla(E(\phi))
$$

hence

$$
-\int_{\Omega}\left(\nabla \cdot \sigma^{e}\right) \cdot \boldsymbol{u}+\int_{\Omega} \boldsymbol{u} \cdot \nabla \phi \frac{\delta E}{\delta \phi}=0 .
$$

Accordingly, the differential problem reads:

$$
\left\{\begin{aligned}
\frac{D \boldsymbol{u}}{D t}-\nabla \cdot \sigma^{d}(D(\boldsymbol{u}), \boldsymbol{n})+\nabla \widetilde{p}-\lambda \frac{\delta E}{\delta \phi} \nabla \phi & =\mathbf{0} & \text { in } \Omega \times(0, T), \\
\nabla \cdot \boldsymbol{u} & =0 & \text { in } \Omega \times(0, T), \\
\frac{D \phi}{D t}+\gamma \frac{\delta E}{\delta \phi} & =0 & \text { in } \Omega \times(0, T),
\end{aligned}\right.
$$

where $\sigma^{d}(D(\boldsymbol{u}), \boldsymbol{n})$ is the symmetric tensor defined in $(2.19), \widetilde{p}=p+\lambda E$ is a modified potential function (that for simplicity it is renamed as $p$ ) and the expression of $\frac{\delta E}{\delta \phi}$ depending on $\phi$ is given in (2.14). This differential problem must be ended with initial conditions

$$
\boldsymbol{u}_{\mid t=0}=\boldsymbol{u}_{0}, \quad \phi_{\mid t=0}=\phi_{0} \quad \text { in } \Omega,
$$


and the non-slip condition $\boldsymbol{u}=0$ on $\partial \Omega \times(0, T)$ and one admissible boundary conditions for $\phi$ given in 2.15). For instance, $\left.\boldsymbol{w} \cdot \boldsymbol{m}\right|_{\partial \Omega}=0$ (that is [N2]) implies the conservation property $\frac{d}{d t} \int_{\Omega} \phi=0$.

Note that the equilibrium solutions $\left(\boldsymbol{u}=0\right.$ and $\phi^{*}$ satisfying $\frac{\delta E}{\delta \phi}\left(\phi^{*}\right)=0$ and the corresponding boundary conditions for $\left.\phi^{*}\right)$ are equilibrium solutions associated to system $(P)$, where the pressure reduces to:

$$
\widetilde{p}=p+\lambda E\left(\phi^{*}\right)=\text { constant }
$$

The fact that system $(P)$ satisfies the (dissipative) energy law (2.23), and therefore the free energy $E_{k i n}(\boldsymbol{u})+\lambda E(\phi)$ has a physical dissipation, play an essential role in the main mathematical results of problem $(P)$, which can be summarized as follows (see Climent-Guillen'13 for a review on mathematical analysis of Nematic and Smectic-A LCs):

- Liu’00] Imposing time-independent Dirichlet boundary conditions for $\phi$, one has

1. existence of global in time weak solutions of $(P)$, satisfying the regularity

$$
\begin{gathered}
\phi \in L^{\infty}\left(0, T ; H^{2}(\Omega)\right) \cap L^{2}\left(0, T ; H^{4}(\Omega)\right), \\
\mathbf{u} \in L^{\infty}\left(0, T ; \boldsymbol{L}^{2}(\Omega)\right) \cap L^{2}\left(0, T ; \boldsymbol{H}^{1}(\Omega)\right),
\end{gathered}
$$

and the energy inequality associated to 2.23$)$ (changing in $(2.23)$ the equality $=0$ by the inequality $\leq 0$ ).

2. existence (and uniqueness) of local in time regular solutions, which is global in time for large viscosity coefficient $\mu_{4}$ (dominant viscosity case),

3. convergence by subsequences at infinite time towards equilibrium solutions $\left(0, \phi^{\star}\right)$ (with $\phi^{\star}$ being a solution of $\left.\frac{\delta E}{\delta \phi}\left(\phi^{\star}\right)=0\right)$.

These results extend the same type of results already obtained for nematic liquid crystal in [Lin-Liu'95, Lin-Liu'00].

- Climent-Guillen'10 Imposing time-dependent Dirichlet conditions for $\phi$, one has existence of time-periodic weak solutions, which are regular for large viscosity $\mu_{4}$.

These results extend the same type of results already obtained for nematic liquid crystal Climent et al.'10, Climent et al.'09.

- Wu-Segatti Imposing periodic boundary conditions for $\phi$, one has convergence to a unique equilibrium solution of the whole sequence $(\boldsymbol{u}(t), \phi(t))$ as time $t \rightarrow+\infty$.

- Climent-Guillen'14 Imposing time-independent Dirichlet conditions for $\phi$, one has convergence to a unique equilibrium solution of the whole sequence $(\boldsymbol{u}(t), \phi(t))$ as time $t \rightarrow+\infty$.

\subsection{Reformulation as a mixed second order problem}

In this section, we derive a new formulation of system $(P)$ that will allow us to consider numerical schemes in the $C^{0}$ finite element framework. For simplicity, we will consider $2 D$ domains and the [D2-N2] boundary conditions for $\phi$, although it is not difficult to extend the same arguments to the $3 D$ case and other admissible boundary conditions for $\phi$, see (2.15) above.

We start introducing a new unknown

$$
\psi:=-\Delta \phi
$$


Hence, we can rewrite the elastic energy in terms of $\psi$, i.e.,

$$
E_{\text {ela }}=\frac{1}{2} \int_{\Omega}(\Delta \phi)^{2} d \boldsymbol{x}=\frac{1}{2} \int_{\Omega} \psi^{2} d \boldsymbol{x} .
$$

Accordingly, we can write the following reformulation of problem $(P)$ for unknowns $(\boldsymbol{u}, p, \phi, \psi)$ :

$(R P)$

$$
\left\{\begin{array}{rll}
\frac{D \boldsymbol{u}}{D t}-\nabla \cdot \sigma^{d}(D(\boldsymbol{u}), \nabla \phi)+\nabla p+\frac{\lambda}{\gamma}\left(\phi_{t}+\boldsymbol{u} \cdot \nabla \phi\right) \nabla \phi & =\mathbf{0} & \text { in } \Omega \times(0, T), \\
\nabla \cdot \boldsymbol{u} & =0 & \text { in } \Omega \times(0, T), \\
\frac{1}{\gamma}\left(\phi_{t}+\boldsymbol{u} \cdot \nabla \phi\right)-\Delta \psi-\frac{1}{\varepsilon^{2}} \nabla \cdot \boldsymbol{f}(\nabla \phi) & =0 & \text { in } \Omega \times(0, T), \\
-\Delta \phi-\psi & =0 & \text { in } \Omega \times(0, T), \\
\boldsymbol{u}=0, \quad \nabla \phi \cdot \boldsymbol{m}=0, \quad\left(\nabla \phi+\frac{1}{\varepsilon^{2}} \boldsymbol{f}(\nabla \phi)\right) \cdot \boldsymbol{m} & =0, & \text { on } \partial \Omega \times(0, T), \\
\boldsymbol{u}_{\mid t=0}=\boldsymbol{u}_{0}, \quad \phi_{\mid t=0} & =\phi_{0} & \text { in } \Omega .
\end{array}\right.
$$

\section{$3 \quad$ Numerical approximations}

In this section, we will introduce a numerical scheme to approximate system $(R P)$. In particular we present a $C^{0}$-finite element approximation in space and a second-order finite difference scheme in time.

\subsection{A generic FEM space-discrete scheme}

Firstly, we will define a space-discrete scheme using $C^{0}$-finite elements. Let $\left\{\mathcal{T}_{h}\right\}_{h>0}$ be a regular triangulations family of $\Omega$ with $h$ denoting the mesh size. Then, the unknowns $(\boldsymbol{u}, p, \phi, \psi)$ are approximated by the conforming finite element spaces:

$$
\left(\boldsymbol{U}_{h}, P_{h}, \Phi_{h}, \Psi_{h}\right) \subset\left(\boldsymbol{H}^{1}(\Omega), L^{2}(\Omega), H^{1}(\Omega), H^{1}(\Omega)\right)
$$

via the following mixed variational formulation of $(R P)$ : Find

$$
(\boldsymbol{u}(t), p(t), \phi(t), \psi(t)) \in \boldsymbol{U}_{h 0} \times P_{h 0} \times \Phi_{h} \times \Psi_{h}
$$

such that

$$
\left\{\begin{aligned}
\left(\boldsymbol{u}_{t}, \overline{\boldsymbol{u}}\right)+c(\boldsymbol{u}, \boldsymbol{u}, \overline{\boldsymbol{u}})+\left(\sigma^{d}(D(\boldsymbol{u}), \nabla \phi), D(\overline{\boldsymbol{u}})\right)-(p, \nabla \cdot \overline{\boldsymbol{u}})+\frac{\lambda}{\gamma}\left(\left(\phi_{t}+\boldsymbol{u} \cdot \nabla \phi\right) \nabla \phi, \overline{\boldsymbol{u}}\right) & =0, \\
(\nabla \cdot \boldsymbol{u}, \bar{p}) & =0, \\
\frac{1}{\gamma}\left(\phi_{t}+\boldsymbol{u} \cdot \nabla \phi, \bar{\phi}\right)+(\nabla \psi, \nabla \bar{\phi})+\frac{1}{\varepsilon^{2}}(\boldsymbol{f}(\nabla \phi), \nabla \bar{\phi}) & =0, \\
(\nabla \phi, \nabla \bar{\psi})-(\psi, \bar{\psi}) & =0,
\end{aligned}\right.
$$

for any $(\overline{\boldsymbol{u}}, \bar{p}, \bar{\phi}, \bar{\psi}) \in \boldsymbol{U}_{h 0} \times P_{h 0} \times \Phi_{h} \times \Psi_{h}$, where $\boldsymbol{U}_{h 0}:=\boldsymbol{U}_{h} \cap \boldsymbol{H}_{0}^{1}(\Omega)$ and $P_{h 0}:=P_{h} \cap L_{0}^{2}(\Omega)$. Hereafter $(\cdot, \cdot)$ denotes the $L^{2}(\Omega)$-scalar product and $c(\cdot, \cdot, \cdot)$ is the trilinear antisymmetric form defined by

$$
c(\boldsymbol{u}, \boldsymbol{v}, \boldsymbol{w})=((\boldsymbol{u} \cdot \nabla) \boldsymbol{v}, \boldsymbol{w})+\frac{1}{2}((\nabla \cdot \boldsymbol{u}) \boldsymbol{v}, \boldsymbol{w}) \quad \forall \boldsymbol{u}, \boldsymbol{v}, \boldsymbol{w} \in \boldsymbol{U}_{h}
$$


On the other hand, from 2.19$)$ and the symmetry of $\sigma^{d}(D(\boldsymbol{u}), \nabla \phi)$, one has

$$
\begin{gathered}
-\left(\nabla \cdot \sigma^{d}(D(\boldsymbol{u}), \nabla \phi), \overline{\boldsymbol{u}}\right)=\left(\sigma^{d}(D(\boldsymbol{u}), \nabla \phi), D(\overline{\boldsymbol{u}})\right) \\
=\mu_{1}\left(\left(\nabla \phi^{t} D(\boldsymbol{u}) \nabla \phi\right),\left(\nabla \phi^{t} D(\overline{\boldsymbol{u}}) \nabla \phi\right)\right)+\mu_{4}(D(\boldsymbol{u}), D(\overline{\boldsymbol{u}}))+2 \mu_{5}(D(\boldsymbol{u}) \nabla \phi, D(\overline{\boldsymbol{u}}) \nabla \phi) .
\end{gathered}
$$

We assume the following inf-sup stability condition:

- For $\left(\boldsymbol{U}_{h 0}, P_{h 0}\right): \sup _{\boldsymbol{u} \in \boldsymbol{U}_{h 0}} \frac{(p, \nabla \cdot \boldsymbol{u})}{\|\boldsymbol{u}\|_{H_{0}^{1}}} \geq \beta_{1}\|p\|_{L^{2}}$ for all $p \in P_{h 0}$.

The following choices could be considered Girault-Raviart'86]:

- $\left(P_{1}\right.$-bubble $) \times \mathrm{P}_{1}$ for $\left(\boldsymbol{U}_{h}, P_{h}\right), \quad \mathrm{P}_{1} \times \mathrm{P}_{1}$ for $\left(\Phi_{h}, \Psi_{h}\right)$.

Lemma 3.1 Each possible solution $(\boldsymbol{u}(t), p(t), \phi(t), \psi(t))$ of the space-discrete scheme (3.3) satisfies the following space-discrete version of the energy law (2.23):

$$
\frac{d}{d t} E_{t o t}(\boldsymbol{u}(t), \nabla \phi(t), \psi(t))+\left(\sigma^{d}(D(\boldsymbol{u}(t)), \nabla \phi(t)), D(\boldsymbol{u}(t))\right)+\frac{\lambda}{\gamma}\left\|\phi_{t}(t)+\boldsymbol{u}(t) \cdot \nabla \phi(t)\right\|_{L^{2}}^{2}=0,
$$

where

$$
E_{\text {tot }}(\boldsymbol{u}, \nabla \phi, \psi):=E_{k i n}(\boldsymbol{u})+\lambda\left(E_{\text {ela }}(\psi)+E_{p e n}(\nabla \phi)\right)
$$

with

$$
E_{k i n}(\boldsymbol{u})=\frac{1}{2}\|\boldsymbol{u}\|_{L^{2}}^{2}, \quad E_{\text {ela }}(\psi)=\frac{1}{2}\|\psi\|_{L^{2}}^{2}, \quad E_{\text {pen }}(\nabla \phi)=\frac{1}{\varepsilon^{2}} \int_{\Omega} F(\nabla \phi) .
$$

Proof. At the initial time we have $\psi(0)=-\Delta \phi(0)$, so we can replace equation $(3.3)_{4}$ by its time derivative

$$
\left(\nabla \phi_{t}, \nabla \bar{\psi}\right)-\left(\psi_{t}, \bar{\psi}\right)=0
$$

and then taking $(\overline{\boldsymbol{u}}, \bar{p}, \bar{\phi}, \bar{\psi})=\left(\boldsymbol{u}(t), p(t), \phi_{t}(t), \lambda \psi(t)\right)$ as test functions in the space-discrete scheme (3.3) and using the equalities

$$
c(\boldsymbol{u}(t), \boldsymbol{u}(t), \boldsymbol{u}(t))=0,
$$

and

$$
\left(\boldsymbol{f}(\nabla \phi(t)), \frac{d}{d t} \nabla \phi(t)\right)=\frac{d}{d t} \int_{\Omega} F(\nabla \phi(t))
$$

one arrives at $(3.6)$.

Remark 3.1 Since

$$
\left(\sigma^{d}(D(\boldsymbol{u}(t)), \nabla \phi(t)), D(\boldsymbol{u}(t))\right) \geq \mu_{4} \int_{\Omega}|D(\boldsymbol{u}(t))|^{2},
$$

from (3.6), one has the following energy inequality

$$
\frac{d}{d t} E_{t o t}(\boldsymbol{u}(t), \nabla \phi(t), \psi(t))+\mu_{4}\|D(\boldsymbol{u}(t))\|_{L^{2}}^{2}+\frac{\lambda}{\gamma}\left\|\phi_{t}(t)+\boldsymbol{u}(t) \cdot \nabla \phi(t)\right\|_{L^{2}}^{2} \leq 0 .
$$




\subsection{Generic second order in time scheme}

We assume an uniform partition of $[0, T]: t_{n}=n k$, with $k=T / N$ denoting the time step. We consider fully coupled second order in time semi-implicit finite difference schemes, defined as:

Given $\left(\boldsymbol{u}^{n}, \phi^{n}, \psi^{n}\right)$, compute $\left(\boldsymbol{u}^{n+1}, p^{n+\frac{1}{2}}, \phi^{n+1}, \psi^{n+\frac{1}{2}}\right) \in \boldsymbol{U}_{h 0} \times P_{h 0} \times \Phi_{h} \times W_{h}$ such that for any $(\overline{\boldsymbol{u}}, \bar{p}, \bar{\phi}, \bar{\psi}) \in \boldsymbol{U}_{h 0} \times P_{h 0} \times \Phi_{h} \times W_{h}:$

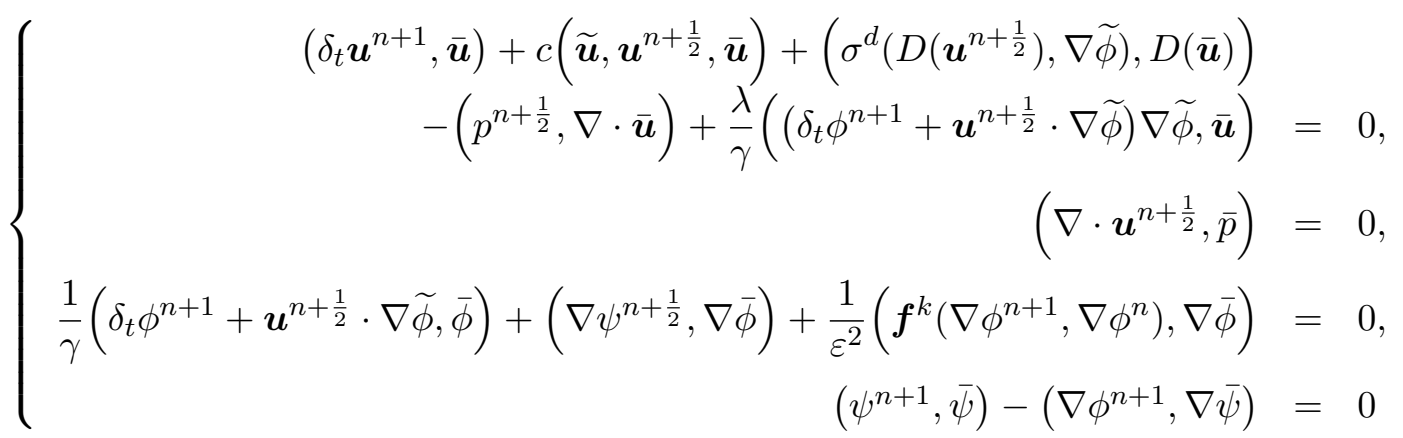

where $\delta_{t}$ denotes the discrete time derivative $\left(\delta_{t} a^{n+1}:=\left(a^{n+1}-a^{n}\right) / k\right)$, unknown $p^{n+\frac{1}{2}}$ is an approximation at midpoint $t^{n+\frac{1}{2}}:=\left(t^{n}+t^{n+1}\right) / 2$ (directly computed), while $\boldsymbol{u}^{n+\frac{1}{2}}:=\left(\boldsymbol{u}^{n+1}+\boldsymbol{u}^{n}\right) / 2$ and $\psi^{n+\frac{1}{2}}:=\left(\psi^{n+1}+\psi^{n}\right) / 2$.

To assure the second order accuracy of the previous scheme, $\boldsymbol{f}^{k}\left(\nabla \phi^{n+1}, \nabla \phi^{n}\right), \widetilde{\boldsymbol{u}}$ and $\nabla \widetilde{\phi}$ have to be defined as second order approximations of $\boldsymbol{f}\left(\nabla \phi\left(t^{n+\frac{1}{2}}\right)\right), \boldsymbol{u}\left(t^{n+\frac{1}{2}}\right)$ and $\nabla \phi\left(t^{n+\frac{1}{2}}\right)$, respectively.

Lemma 3.2 The following discrete energy inequality holds:

$$
\delta_{t} E_{t o t}\left(\boldsymbol{u}^{n+1}, \nabla \phi^{n+1}, \psi^{n+1}\right)+\mu_{4}\left\|D\left(\boldsymbol{u}^{n+\frac{1}{2}}\right)\right\|_{L^{2}(\Omega)}^{2}+\frac{\lambda}{\gamma}\left\|\delta_{t} \phi^{n+1}+\boldsymbol{u}^{n+\frac{1}{2}} \cdot \nabla \widetilde{\phi}\right\|_{L^{2}(\Omega)}^{2}+\frac{\lambda}{\varepsilon^{2}} N D_{\text {phobic }}^{n+1} \leq 0
$$

where

$$
N D_{\text {phobic }}^{n+1}=\int_{\Omega} f^{k}\left(\nabla \phi^{n+1}, \nabla \phi^{n}\right) \cdot \delta_{t} \nabla \phi^{n+1}-\delta_{t}\left(\int_{\Omega} F\left(\nabla \phi^{n+1}\right)\right) .
$$

Proof. Considering $\psi^{0}=P_{\Psi_{h}}^{L^{2}}\left(-\Delta \phi^{0}\right.$ ) (where $P_{\Psi_{h}}^{L^{2}}$ denotes the $L^{2}$-projection onto $\Psi_{h}$ ), by induction and using from the previous time step that

$$
\left(\psi^{n}, \bar{\psi}\right)-\left(\nabla \phi^{n}, \nabla \bar{\psi}\right)=0,
$$

we can replace equation $(3.10)_{5}$ by its discrete time derivative

$$
\left(\delta_{t} \psi^{n+1}, \bar{\psi}\right)-\left(\delta_{t} \phi^{n+1}, \nabla \bar{\psi}\right)=0 .
$$

Then, taking as test functions in the scheme (3.10)

$$
(\overline{\boldsymbol{u}}, \bar{p}, \bar{\phi}, \bar{\psi})=\left(\boldsymbol{u}^{n+\frac{1}{2}}, p^{n+\frac{1}{2}}, \lambda \delta_{t} \phi^{n+1}, \lambda \psi^{n+\frac{1}{2}}\right),
$$

the term $\left(\delta_{t} \phi^{n+1}, \nabla \psi^{n+\frac{1}{2}}\right)$ cancels, and by using the expressions

$$
c\left(\widetilde{\boldsymbol{u}}, \boldsymbol{u}^{n+\frac{1}{2}}, \boldsymbol{u}^{n+\frac{1}{2}}\right)=0,
$$

and

$$
\left(\sigma^{d}\left(D\left(\boldsymbol{u}^{n+\frac{1}{2}}\right), \nabla \widetilde{\phi}\right), D\left(\boldsymbol{u}^{n+\frac{1}{2}}\right)\right) \geq \mu_{4} \int_{\Omega}\left|D\left(\boldsymbol{u}^{n+\frac{1}{2}}\right)\right|^{2},
$$

one arrives at (3.11). 
Definition 3.1 The scheme (3.10) is energy-stable if it holds

$$
\delta_{t} E_{t o t}\left(\boldsymbol{u}^{n+1}, \nabla \phi^{n+1}, \psi^{n+1}\right)+\mu_{4}\left\|D\left(\boldsymbol{u}^{n+\frac{1}{2}}\right)\right\|_{L^{2}(\Omega)}^{2}+\frac{\lambda}{\gamma}\left\|\delta_{t} \phi^{n+1}+\boldsymbol{u}^{n+\frac{1}{2}} \cdot \nabla \widetilde{\phi}\right\|_{L^{2}(\Omega)}^{2} \leq 0 .
$$

In particular, energy-stable schemes satisfy the energy decreasing in time property,

$$
E_{t o t}\left(\boldsymbol{u}^{n+1}, \nabla \phi^{n+1}, \psi^{n+1}\right) \leq E_{t o t}\left(\boldsymbol{u}^{n}, \nabla \phi^{n}, \psi^{n}\right), \quad \forall n .
$$

In particular, depending on the approximation considered of $f^{k}\left(\nabla \phi^{n+1}, \nabla \phi^{n}\right)$ we will obtain different numerical schemes, with different discrete energy laws. We refer the reader to [Tierra-Guillen'14] for more detailed information about different ways of efficient handling this term.

Lemma 3.3 (Global energy-stability) Assuming the scheme (3.10) is energy-stable, then the following estimates hold:

$$
\boldsymbol{u}^{n+1} \text { in } l^{2}\left(0, T ; \boldsymbol{H}_{0}^{1}\right), \quad \psi^{n+1} \text { in } l^{\infty}\left(0, T ; \boldsymbol{L}^{2}\right), \quad \text { and } \nabla \phi^{n+1} \text { in } l^{4}\left(0, T ; \boldsymbol{L}^{4}\right) .
$$

Proof. By induction respect to the time step $n,(3.14$ implies the following bounds independent of $k, h, \varepsilon$ (if $\left|\boldsymbol{u}^{0}\right|^{2}+\left|\psi^{0}\right|^{2} \leq C$ and $\int_{\Omega} F\left(\nabla \phi^{0}\right) \leq C \varepsilon^{2}$, with $C>0$ independent of $k, h$ and $\varepsilon$ ):

$$
E_{t o t}\left(\boldsymbol{u}^{n+1}, \nabla \phi^{n+1}, \psi^{n+1}\right) \leq C, \quad k \sum_{n}\left(\left\|D\left(\boldsymbol{u}^{n+1}\right)\right\|_{L^{2}(\Omega)}^{2}+\left\|\delta_{t} \phi^{n+1}+\boldsymbol{u}^{n+\frac{1}{2}} \cdot \nabla \widetilde{\phi}\right\|_{L^{2}(\Omega)}^{2}\right) \leq C,
$$

hence the following $(k, h, \varepsilon)$-independent estimates hold

$$
\begin{array}{cll}
\boldsymbol{u}^{n+1} \text { and } \psi^{n+1} & \text { in } \quad l^{\infty}\left(0, T ; \boldsymbol{L}^{2}\right), & \frac{1}{\varepsilon^{2}} \int_{\Omega} F\left(\nabla \phi^{n+1}\right) \quad \text { in } \quad l^{\infty}(0, T), \\
D\left(\boldsymbol{u}^{n+1}\right) & \text { in } \quad l^{2}\left(0, T ; L^{2}\right) .
\end{array}
$$

In particular, by applying Korn's inequality to $\boldsymbol{u}^{n+1}$, one also has the $(k, h, \varepsilon)$-independent estimate

$$
\boldsymbol{u}^{n+1} \text { in } l^{2}\left(0, T ; \boldsymbol{H}_{0}^{1}\right) .
$$

On the other hand, owing to the inequality $\int_{\Omega} F(\nabla \phi) \geq\left(\|\nabla \phi\|_{L^{4}(\Omega)}^{4}-|\Omega|\right)$, one also has the following $(k, h)$-independent estimate

$$
\nabla \phi^{n+1} \text { is bounded in } l^{4}\left(0, T ; \boldsymbol{L}^{4}\right),
$$

although this bound depends on $\varepsilon$.

\subsubsection{How to define $\boldsymbol{f}^{k}\left(\nabla \phi^{n+1}, \nabla \phi^{n}\right)$}

There are several possible ways of approximating potential $\boldsymbol{f}(\nabla \phi)$ (check Tierra-Guillen'14] for a detailed review). Indeed, in the last years many works from different physical applications have appeared in the literature, presenting new ways of dealing with this kind of potentials. For the Cahn-Hilliard equation, implicit approximations have been often considered [Elliot-French'89, Elliot-French'87, Elliot-French-1 Feng-Prohl'04, Copetti-Elliot'92, Gomez et al.'08, where a Newton method is usually employed in order to compute the nonlinear scheme. There is an implicit-explicit approximation of the potential that does not introduce any numerical dissipation which have been widely used in phase field models Du-Nicolaides'91, Feng'06, Hua et al.'11, Hyon-Kwak-Liu'10 and in the Liquid Crystals context Lin-Liu-Zhang'07. On the other hand, many authors split the potential into a convex and a nonconvex part in order to assure the existence of some numerical dissipation to obtain a unconditional 
energy-stable scheme Eyre, Becker-Feng-Prohl'08, Hu et al.'09, Kim-Kang-Lowengrub'04, Wise'10, Mello-Filho'05, Gomez-Hughes'11, although the resulting schemes are nonlinear. The idea of splitting the potential has been also considered for thin film epitaxy Shen et al.'12. Moreover, some linear schemes have been studied in Shen-Yang'10, Badia-Guillén-Gutiérrez'11, Guillen-Tierra'13, Guillen-Tierra'14, Wu-van Zwieten-van der Zee'13].

Solvability of scheme 3.10$)$ will depend on the approximation of the potential term $\boldsymbol{f}^{k}\left(\nabla \phi^{n+1}, \nabla \phi^{n}\right)$. In the case of energy-stable schemes (non-linear schemes), solvability follows from the discrete energy law (3.11) and either an application of the Brouwer fixed-point theorem (cf. Corollary 1.1 of Girault-Raviart'86]) or an application of the Leray-Schauder fixed-point theorem in a finite dimensional setting. But, the uniqueness of these nonlinear schemes is not clear in general, depending on the behavior of

$$
\frac{\lambda}{\varepsilon^{2} k}\left(\boldsymbol{f}^{k}\left(\nabla \phi^{n+1}, \nabla \phi^{n}\right)-\boldsymbol{f}^{k}\left(\nabla \widetilde{\phi}^{n+1}, \nabla \phi^{n}\right), \nabla \phi^{n+1}-\nabla \widetilde{\phi}^{n+1}\right)
$$

where $\nabla \phi^{n+1}$ and $\nabla \widetilde{\phi}^{n+1}$ are two possible solutions of the scheme. In fact, using a convex-concave semi-implicit approximation (assuming implicitly the convex part and explicitly the concave one) the uniqueness is deduced from the monotony of $f^{k}\left(\cdot, \nabla \phi^{n}\right)$ (because the convex part of the potential is treated implicitly). In the case of the concave part treated implicitly, one can not deduce unconditional uniqueness and a constraint of time step $k$ small enough must be imposed.

\subsubsection{How to define $\widetilde{\boldsymbol{u}}$ and $\nabla \widetilde{\phi}$}

The scheme 3.10 has been designed as second order approximations of the associated model, but it is necessary to define $\widetilde{\boldsymbol{u}}$ and $\nabla \widetilde{\phi}$ as second order approximations of $\boldsymbol{u}\left(t^{n+\frac{1}{2}}\right)$ and $\boldsymbol{n}\left(t^{n+\frac{1}{2}}\right)$, respectively. We propose two different ways of dealing with these terms:

1. The first one consists on choosing a Crank-Nicolson approach,

$$
\widetilde{\boldsymbol{u}}=\left(\boldsymbol{u}^{n+1}+\boldsymbol{u}^{n}\right) / 2 \quad \text { and } \quad \nabla \widetilde{\phi}=\left(\nabla \phi^{n+1}+\nabla \phi^{n}\right) / 2 .
$$

In this case we obtain a nonlinear one-step scheme.

2. The second way consists on using a BDF2 approximation for each one of the unknowns, i.e.,

$$
\widetilde{\boldsymbol{u}}=\left(3 \boldsymbol{u}^{n}-\boldsymbol{u}^{n-1}\right) / 2 \quad \text { and } \quad \nabla \widetilde{\phi}=\left(3 \nabla \phi^{n}-\nabla \phi^{n-1}\right) / 2 .
$$

Then, using these two-step approximations, the linearity and the solvability conditions of the schemes will depend just on the choice of the potential term $f^{k}\left(\nabla \phi^{n+1}, \nabla \phi^{n}\right)$.

\section{Numerical simulations}

In this section we study the behavior of the numerical schemes presented through the paper. In particular, we will focus on the second order scheme obtained using the generic scheme (3.10) with the linear potential approximation OD2 (whose efficiency have been studied for the Cahn-Hilliard equation in [Tierra-Guillen'14]):

$$
\boldsymbol{f}^{k}\left(\nabla \phi^{n+1}, \nabla \phi^{n}\right)=\boldsymbol{f}\left(\nabla \phi^{n}\right)+\frac{1}{2} \boldsymbol{f}^{\prime}\left(\nabla \phi^{n}\right)\left(\nabla \phi^{n+1}-\nabla \phi^{n}\right),
$$

that it is a second order in time approximation of the potential $\boldsymbol{f}\left(\nabla \phi\left(t^{n+\frac{1}{2}}\right)\right)$. In particular, for the Ginzburg-Landau potential $\boldsymbol{f}(\nabla \phi)$ considered in this paper, a direct computation yields to

$$
\boldsymbol{f}^{k}\left(\nabla \phi^{n+1}, \nabla \phi^{n}\right)=\left(\nabla \phi^{n} \cdot \nabla \phi^{n+1}\right) \nabla \phi^{n}+\left|\nabla \phi^{n}\right|^{2} \frac{\nabla \phi^{n+1}-\nabla \phi^{n}}{2}-\frac{\nabla \phi^{n+1}+\nabla \phi^{n}}{2} .
$$


By using ideas from Guillen-Tierra'13, Guillen-Tierra'14, the unique solvability of the scheme (3.10) using the approximation (4.2) can be assured under the constraint $k<2 \varepsilon^{2} / \gamma$. Nevertheless, energystability of scheme (3.10) and (4.2) (possibly assuming constraints of type $k$ small enough in function of $h$ and $\varepsilon$ ), remain as an open problem.

In order to complete a fully linear scheme, we take the linear BDF2 approximations presented in (3.18).

The domain considered is $\Omega=(-1,1)^{2}$ with an uniform space partition of size $h=1 / 32$, the time step set to $k=10^{-5}$ and the physical parameters set to $\mu_{1}=\mu_{4}=\mu_{5}=\lambda=\gamma=1$ and $\varepsilon=0.05$. A finite element approximation is considered in space, using the software FreeFem ++ Hetch'12 for carrying out the simulations under the following choices for the discrete spaces:

$$
(\boldsymbol{u}, p) \sim P_{2} \times P_{1} \quad \text { and } \quad(\phi, \psi) \sim P_{1} \times P_{1} .
$$

We consider the initial conditions

$$
\phi(0)=\sin (x) \cos (y)^{2}, \quad \psi(0)=-\Delta \phi(0), \quad \boldsymbol{u}_{1}(0)=0, \quad \boldsymbol{u}_{2}(0)=0,
$$

the boundary conditions

$$
\boldsymbol{u}=\nabla \phi \cdot \boldsymbol{m}=\left(\nabla \psi+\frac{1}{\varepsilon^{2}} \boldsymbol{f}(\nabla \phi)\right) \cdot \boldsymbol{m}=0 \quad \text { on } \partial \Omega
$$

(which correspond to [D2-N2] in (2.15) ) and we compute a first step $\left(\boldsymbol{n}^{1}, \boldsymbol{u}^{1}\right)$ using the scheme (3.10) with the Crank-Nicolson approximation described in (3.17), i.e.,

$$
\widetilde{\boldsymbol{u}}=\left(\boldsymbol{u}^{n+1}+\boldsymbol{u}^{n}\right) / 2 \quad \text { and } \quad \widetilde{\boldsymbol{n}}=\left(\boldsymbol{n}^{n+1}+\boldsymbol{n}^{n}\right) / 2,
$$

in order to be able to use the BDF2 approximation for $\widetilde{\boldsymbol{u}}$ and $\widetilde{\boldsymbol{n}}$ in the rest of time iterations.

The dynamics of the layer $\phi$ and the velocity field $\boldsymbol{u}$ are presented in Figures $4.1+4.2$ while in Figures 4.34 .4 we plot the evolution of the kinetic and elastic energy. The dynamics consists on the deformation of the layer configuration to reach an equilibrium configuration, that induces movement in the fluid part. Moreover, the total energy dissipates until there are no changes on the layer and on the velocity field, i.e., the system is reaching an equilibrium configuration. 

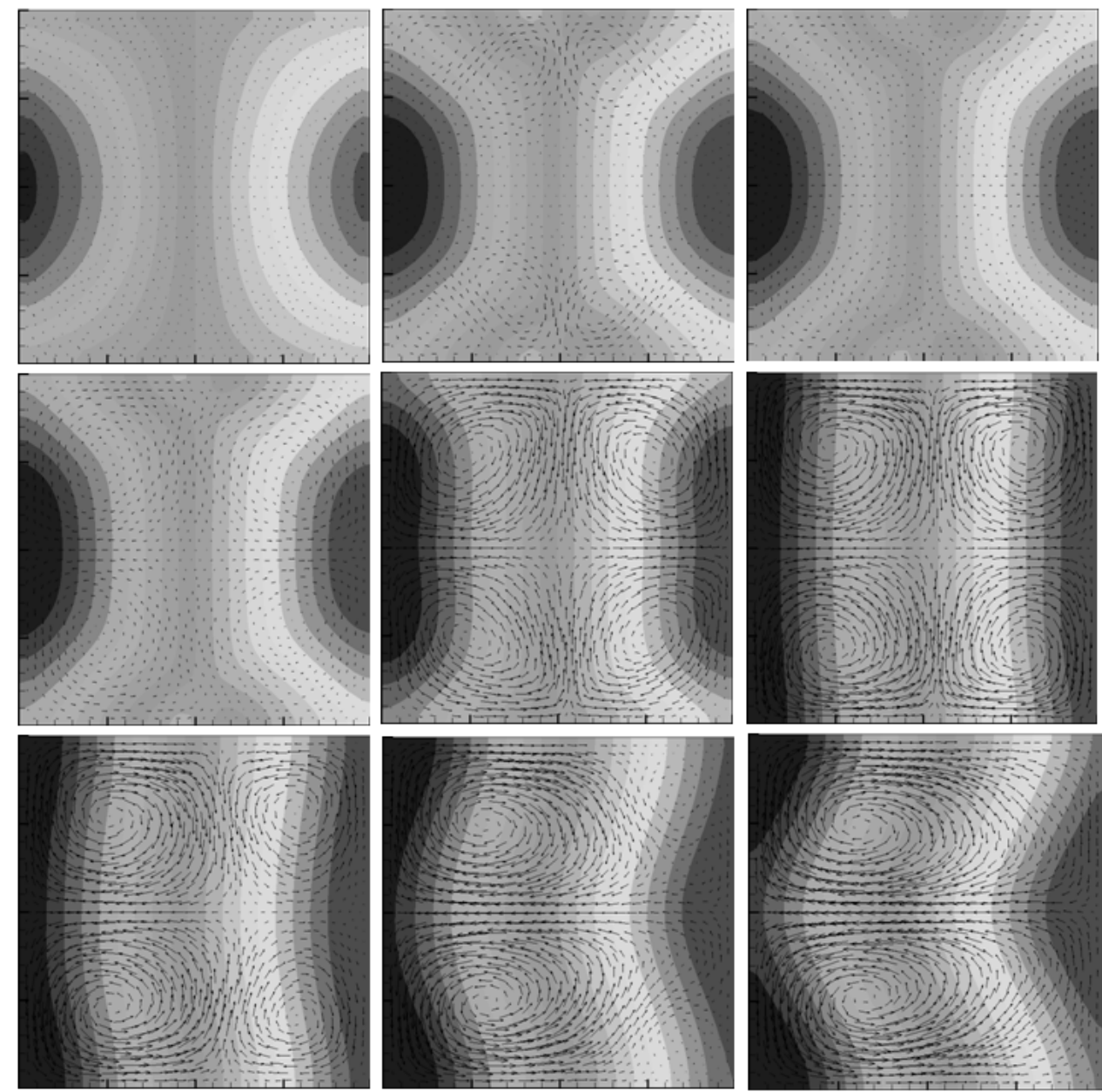

Figure 4.1: From left to right and up to down, the evolution of $\phi$ in time $t=$ $0.00001,0.005,0.01,0.015,0.02,0.025,0.03,0.035$ and 0.04 

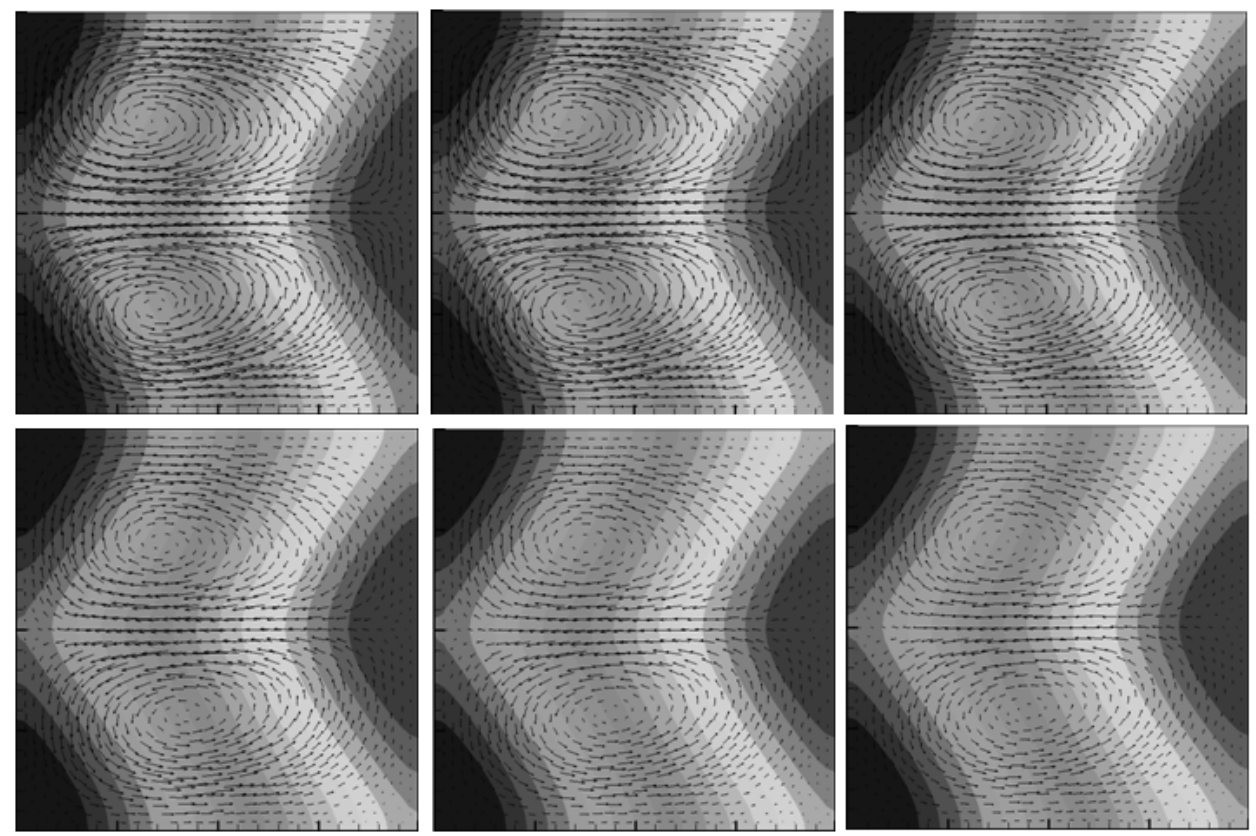

Figure 4.2: From left to right and up to down, the evolution of $\phi$ in time $t=0.045,0.05,0.06,0.07,0.08$ and 0.086

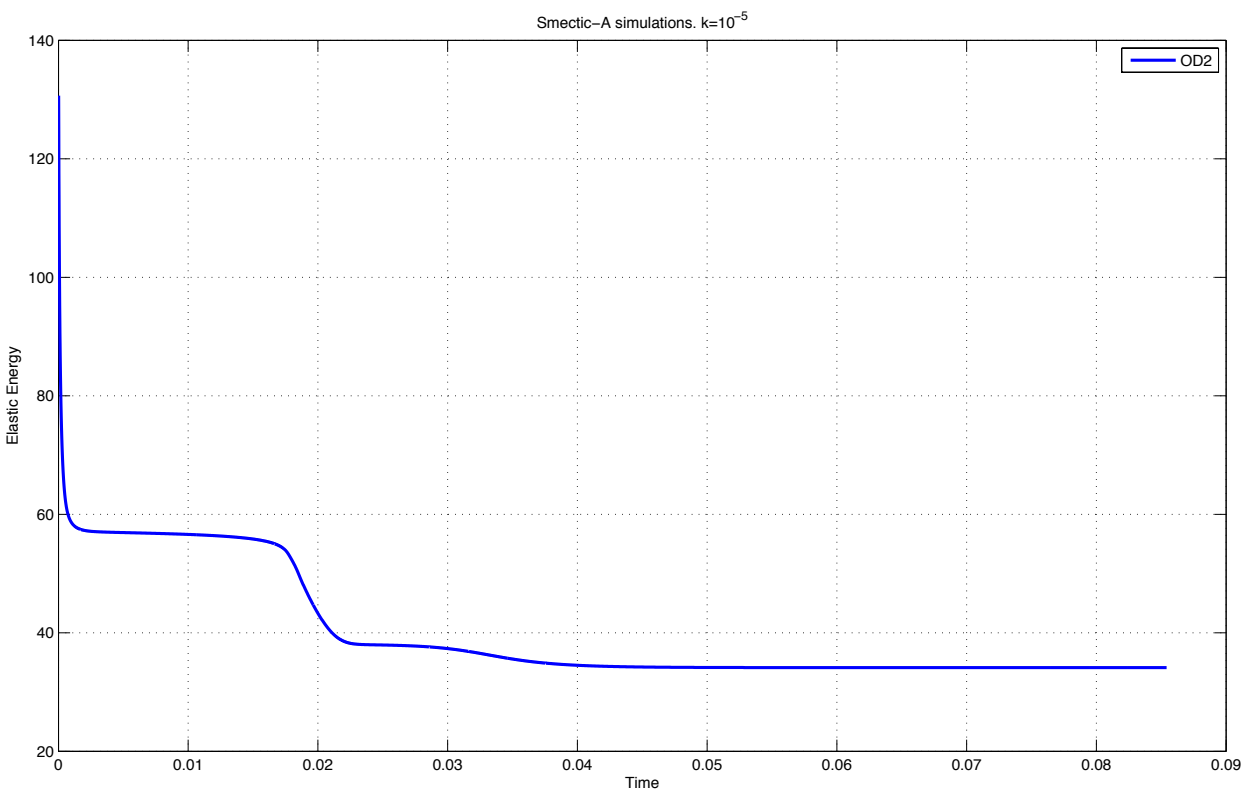

Figure 4.3: Kinetic energy 


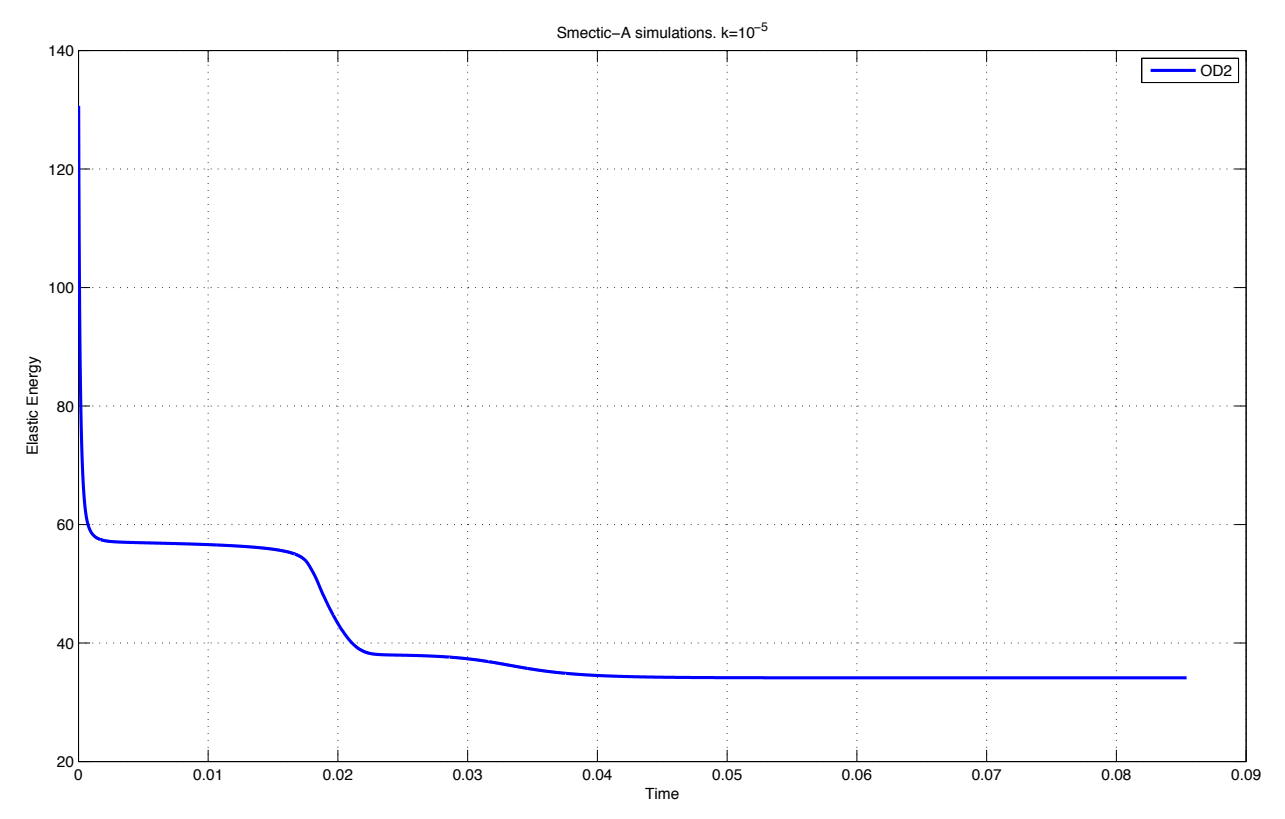

Figure 4.4: Elastic energy

\section{Conclusions}

In this paper, we have presented a new reformulation of the Smectic-A liquid crystals system, where a new unknown $\psi=-\Delta \phi$ have been introduced in order to arrive at a mixed second-order problem. This new formulation allows us to recover a dissipative energy law, that is in correspondence with the energy law associated to the original problem.

We approximate this new formulation using second-order finite differences in time and by $C^{0}$-finite elements in space. For this scheme, we deduce a discrete version of the dissipative energy law derived in the continuous problem.

Finally, numerical simulations are reported to show that the proposed scheme capture the dynamics of Smectic-A liquid crystals. More extensive numerical tests and studies of other effects, such as the influence of physical parameters and the interaction with other type of fluids will be also investigated in the future.

\section{Acknowledgements.}

This research has been partially supported by MTM2012-32325 (Ministerio de Economía y Competitividad, Spain). Giordano Tierra has also been partially supported by ERC-CZ project LL1202 (Ministry of Education, Youth and Sports of the Czech Republic).

\section{References}

[Badia-Guillén-Gutiérrez'11] S. Badia, F. Guillén-González, J.V. Gutiérrez-Santacreu; An Overview on Numerical Analyses of Nematic Liquid Crystal Flows, Archives of Computational Methods in Engineering, 18 (2011) 285-313 
[Badia-Guillén-Gutiérrez'11] Badia, S.; Guillén-González, F.; Gutiérrez-Santacreu, J.V.; Finite element approximation of nematic liquid crystal flows using a saddle-point structure, Journal of Computational Physics 230 (2011) 1686-1706.

[Becker-Feng-Prohl'08] R. Becker, X. Feng, A. Prohl. Finite element approximations of the EricksenLeslie model for nematic liquid crystal flow, SIAM Journal Numer. Anal. 46 (2008) 1704-1731.

[Climent-Guillen'13] B. Climent-Ezquerra, F. Guillén-González. A review of mathematical analysis of nematic and smectic-A liquid crystal models, European Journal of Applied Mathematics 10 (2013) 1-21.

[Climent-Guillen'14] B. Climent-Ezquerra, F. Guillén-González. Convergence to equilibrium for smectic-A liquid crystals in $3 D$ domains without constraints for the viscosity, Nonlinear Analysis Theory, Methods and Applications 102 (2014) 208-219.

[Climent-Guillen'10] B. Climent-Ezquerra, F. Guillén-González. Global in time solution and timeperiodicity for a smectic-A liquid crystal model, Commun. Pure Appl. Anal. 9 (2010) 1473-1493.

[Climent et al.'09] B. Climent-Ezquerra, F. Guillén-González, M.J. Moreno-Iraberte. Regularity and Time-periodicity for a Nematic Liquid Crystal model, Nonlinear Analysis Theory, Methods and Applications, 71 (2009) 539-549

[Climent et al.'10] B. Climent-Ezquerra, F. Guillén-González, M.A. Rodríguez Bellido. Stability for Nematic Liquid Crystals with stretching terms, International Journal of Bifurcations and Chaos, 20 (2010) 2937-2942.

[Collings'90] P.J. Collings. Liquid Crystals: Nature's Delicate Phase of Matter, Princeton University Press (1990)

[Copetti-Elliot'92] Copetti, M.I.M.; Elliott, C.M.; Numerical analysis of the Cahn-Hilliard equation with a logarithmic free energy. Numer. Math. 63 (1992) 39-65.

[Coutand-Shkoller'01] D. Coutand, S. Shkoller. Well posedness of the full Ericsen-Leslye model of nematic liquid crystals, C. R. Acad. Sci. Paris, 333 (2001) 919-924.

[Du-Nicolaides'91] Du, Q.; Nicolaides, R.A.; Numerical analysis of a continuum model of phase transition. SIAM Journal Numer. Anal. 28 (1991) 1310-1322.

[E'97] W. E. Nonlinear Continuum Theory of Smectic-A Liquid Crystals, Arch. Rat. Mech. Anal., 137 (1997) 159-175.

[Elliot-French'89] Elliott C.M., French D.A. A nonconforming finite-element method for the twodimensional Cahn-Hilliard equation, SIAM Journal Numer. Anal. 26 (1989) 884-903.

[Elliot-French'87] Elliott C.M., French D.A. Numerical studies of the Cahn-Hilliard equation for phase separation, IMA Journal Applied Math. 38 (1987) 97-128.

[Elliot-French-Milner'89] Elliott, C.M.; French, D.A.; Milner, F.A.; A Second Order Splitting Method for the Cahn-Hilliard Equation, Numer. Math. 54 (1989) 575-590.

[Eyre] Eyre, J.D.; An Unconditionally Stable One-Step Scheme for Gradient System, unpublished, www.math.utah.edu/ eyre/research/methods/stable.ps 
[Feng'06] Feng, X.; Fully discrete finite element approximations of the Navier-Stokes-Cahn-Hilliard diffuse interface model for two-phase fluid flows, SIAM J. Numer. Anal. 44 (2006) 1049-1072.

[Feng-Prohl'04] Feng, X.; Prohl, A.; Error analysis of a mixed finite element method for the CahnHilliard equation, Numer. Math. 99 (2004) 47-84.

[Girault-Raviart'86] V. Girault, P.A. Raviart. Finite element methods for Navier-Stokes equations: theory and algorithms. Berlin, Springer-Verlag, 1986.

[Gomez et al.'08] Gomez, H.; Calo, V.M.; Bazilevs, Y.; Hughes, T.J.R.; Isogeometric analysis of the Cahn-Hilliard phase-field model, Comput. Methods Appl. Mech. Engrg. 197 (2008) 4333-4352.

[Gomez-Hughes'11] Gomez, H.; Hughes, T.J.R.; Provably Unconditionally Stable, Second-order Timeaccurate, Journal of Computational Physics 230 (2011) 5310-5327.

[Guillen-Tierra'13] Guillén-González, F.; Tierra, G.; On linear schemes for a Cahn Hilliard Diffuse Interface Model. J. Comput Physics. 234 (2013) 140-171.

[Guillen-Tierra'14] Guillén-González, F.; Tierra, G.; Second order schemes and time-step adaptivity for Allen-Cahn and Cahn-Hilliard models. Submitted.

[Hetch'12] Hecht, F. New development in freefem++, J. Numer. Math., 20 (2012) 251-265

[Hu et al.'09] Hu Z., Wise S.M., Wang C., Lowengrub J.S. Stable and efficient finite-difference nonlinear-multigrid schemes for the phase field crystal equation, Journal of Computational Physics 228 (2009) 5323-5339

[Hua et al.'11] Hua, J.; Lin. P.; Liu, C.; Wang, Q.; Energy law preserving C0 finite element schemes for phase field models in two-phase flow computations, Journal of Computational Physics 230 (2011) 7115-7131.

[Hyon-Kwak-Liu'10] Hyon Y.; Kwak D.Y.; Liu C; Energetic variational approach in complex fluids: Maximum dissipation principle, Discrete and Continuous Dynamical Systems 26 (2010) 12911304.

[Kim-Kang-Lowengrub'04] Kim J.; Kang K.; Lowengrub J.; Conservative multigrid methods for CahnHilliard fluids, Journal of Computational Physics 193 (2004) 511-543.

[Lin'89] F.H. Lin. Nonlinear theory of defects in nematic liquid crystals: phase transition and flow phenomena, Comm. Pure Appl. Math., 42 (1989) 789-814.

[Lin-Liu'95] F.H. Lin, C. Liu. Non-parabolic dissipative systems modelling the flow of liquid crystals, Comm. Pure Appl. Math., 4 (1995) 501-537.

[Lin-Liu'00] F.H. Lin, C. Liu. Existence of solutions for the Ericksen-Leslie system, Arch. Rat. Mech. Anal., 154 (2000) 135-156.

[Lin-Liu-Zhang'07] Lin, P.; Liu, C.; Zhang, H.; An energy law preserving $C^{0}$ finite element scheme for simulating the kinematic effects in liquid crystal dynamics, Journal of Computational Physics 227 (2007) 1411-1427.

[Liu'00] C. Liu. Dynamic Theory for Incompressible Smectic Liquid Crystals: Existence and Regularity, Discret Contin Dyn S , 6 (2000) 591-608. 
[Mello-Filho'05] Mello, E.V.L.; Filho, O.T.S.; Numerical study of the Cahn-Hilliard equation in one, two and three dimensions, Physica A 347 (2005) 429-443.

[Shen et al.'12] Shen, J.; Wang, C.; Wang, X.; Wise, S.; Second-order convex splitting schemes for gradient flows with Enhrich-Schwoebel type energy: application to thin film epitaxy, SIAM J. Numer. Anal. 50 (2012)105-125

[Shen-Yang'10] Shen, J.; Yang, X.; Numerical approximations of Allen-Cahn and Cahn-Hilliard equations, Discrete and continuous dynamical systems 28 (2010) 1669-1691.

[Tierra-Guillen'14] Tierra, G.; Guillén-González, F.; Numerical methods for solving the Cahn-Hilliard equation and its applicability to related Energy-based models. Archives of Computational Methods in Engineering. DOI:10.1007/s11831-014-9112-1

[Wise'10] Wise S.M.; Unconditionally Stable Finite Difference, Nonlinear Multigrid Simulation of the Cahn-Hilliard-Hele-Shaw System of Equations, Journal of Scientific Computing 441 (2010).

[Wu-Segatti] A. Segatti, H. Wu. Finite dimensional reduction and convergence to equilibrium for incompressible Smectic-A liquid crystal flows, SIAM J. Math. Anal., 43 (2011) 2445-2481.

[Wu-van Zwieten-van der Zee'13] Wu, X.; van Zwieten, G.J.; van der Zee, K.G.; Stabilized secondorder convex splitting schemes for Cahn-Hilliard models with application to diffuse-interface tumor-growth models, Int. J. Numer. Meth. Biomed. Engng. 228 (2013) DOI: 10.1002/cnm.2597 